\title{
APLICAÇÃO DO MÉTODO DE ENSAIO DAS FREQUÊNCIAS NATURAIS DE VIBRAÇÃ̃O PARA OBTENÇÃO DO MÓDULO DE ELASTICIDADE DE PEÇAS ESTRUTURAIS DE MADEIRA ${ }^{1}$
}

\author{
Pedro Gutemberg de Alcântara Segundinho ${ }^{2}$, Leiliane Cristina Cossolino ${ }^{3}$, Antônio Henrique Alves \\ Pereira $^{3}$ e Carlito Calil Junior ${ }^{4}$
}

\begin{abstract}
RESUMO - Existem diversas técnicas para caracterização do módulo de elasticidade de madeiras e, dentre as atualmente empregadas, destacam-se aquelas que utilizam as frequências naturais de vibração, por serem técnicas não destrutivas e, portanto, apresentarem resultados que podem ser repetidos e comparados ao longo do tempo. Este trabalho teve como objetivo avaliar a eficácia, dos métodos de ensaios baseados nas frequências naturais de vibração comparando-os aos resultados obtidos na flexão estática na obtenção das propriedades elásticas em peças estruturais de madeira de reflorestamento que são usualmente empregadas na construção civil. Foram avaliadas 24 vigas de Eucalyptus sp. com dimensões nominais ( 40 x 60 x $2.000 \mathrm{~mm}$ ) e 14 vigas de Pinus oocarpa com dimensões nominais ( $45 \times 90 \times 2.300 \mathrm{~mm}$ ), ambas sem tratamento; 30 pranchas com dimensões nominais $(40 \times 240 \times 2.010 \mathrm{~mm})$ e 30 pranchas com dimensões nominais $(40 \times 240 \times 3.050 \mathrm{~mm})$, ambas de Pinnus oocarpa e com tratamento preservativo à base de Arseniato de Cobre Cromatado - CCA. Os resultados obtidos apresentaram boa correlação quando comparados aos resultados obtidos pelo método mecânico de flexão estática, especialmente quando empregada a frequência natural de vibração longitudinal. O emprego da frequência longitudinal mostrou-se confiável e prático, portanto recomendada para a determinação do módulo de elasticidade de peças estruturais de madeira. Verificou-se ainda que, empregando a frequência longitudinal, não há necessidade de um suporte específico para os corpos de prova ou calibrações prévias, reduzindo assim o tempo de execução e favorecendo o ensaio de grande quantidade de amostras.
\end{abstract}

Palavras-chave: Ensaios não destrutivos, Frequências naturais de vibração e Módulo de elasticidade.

\section{ANALYSIS OF THE NATURAL VIBRATION FREQUENCY TEST METHOD TO OBTAIN THE MODULUS OF ELASTICITY OF WOOD STRUCTURAL COMPONENTS}

\begin{abstract}
There are several techniques to characterize the elastic modulus of wood and those currently using the natural frequencies of vibration stand out as they are non-destructive techniques, producing results that can be repeated and compared over time. This study reports on the effectiveness of the testing methods based on the natural frequencies of vibration versus static bending to obtain the elastic properties of reforested structural wood components usually employed in civil construction. The following components were evaluated: 24 beams of Eucalyptus sp. with nominal dimensions $(40 \times 60 \times 2.000 \mathrm{~mm})$ and 14 beams of Pinus oocarpa with nominal dimensions ( $45 \times 90 \times 2.300 \mathrm{~mm}$ ) both without treatment; 30 boards with nominal dimensions $(40 \times 240 \times 2.010 \mathrm{~mm})$ and 30 boards with nominal dimensions $(40 \times 240 \times 3.050 \mathrm{~mm})$, both of Pinus oocarpa and with chromate copper arsenate (CCA) preservative treatment. The results obtained in thiswork show good correlation when compared to the results obtained by the static bending mechanical method, especially when
\end{abstract}

\footnotetext{
${ }^{1}$ Recebido em 02.07.2011 aceito para publicação em 04.06.2012

${ }^{2}$ Universidade Federal do Espírito Santo, Centro de Ciências Agrárias, Departamento de Ciências Florestais e da Madeira, Jerônimo Monteiro, ES, Brasil. E-mails: <p_gutemberg2001@yahoo.com.br>e <pedro.segundinho@ufes.br>.

${ }^{3}$ ATCP Engenharia Física, São Carlos, SP, Brasil. E-mails: <apl@atcp.com.br $>$ e $<$ ha @ atcp.com.br $>$.

${ }^{4}$ Universidade de São Paulo, Escola de Engenharia de São Carlos, Departamento de Engenharia de Estruturas, São Carlos, SP, Brasil.E-mail: <calil@sc.usp.br>.
} 
applying the natural frequency of longitudinal vibration. The use of longitudinal frequency was reliable and practical, therefore recommended for determining the modulus of elasticity of wood structural elements. It was also found that no specific support is needed for the specimens using the longitudinal frequency, as well as no previous calibrations, reducing the execution time and enabling to test many samples.

Keywords: Non-destructive tests, Natural frequencies of vibration and Modulus of elasticity.

\section{INTRODUÇÃO}

Tal como acontece com todos os materiais utilizados na construção civil, a madeira para uso estrutural deve ser classificada pelas suas propriedades físicas e mecânicas. Atualmente, a norma brasileira NBR 7190 (1997) estabelece o sistema de classes de resistência para seu uso, isto é, a norma prescreve ao engenheiro possíveis classes de resistência, associando a cada uma delas sua resistência característica, módulo de elasticidade e densidade. No projeto de revisão da NBR 7190 que se encontra em análise, a partir de 2011 foram incluídas regras de classificação visual para distribuição da madeira em lotes de uso estrutural, conforme sugerido por Carreira e Dias (2005), a partir da qual poderá ser feita a classificação mecânica. Dessa forma, o processo inicia-se por meio de métodos não destrutivos, em que é feita uma inspeção visual para examinar algumas características intrínsecas (inclinação das fibras, nós, empenamento, anéis de crescimento, encurvamento, racha anelar e fenda), agrupando-as em lotes menos heterogêneos (FIORELLI et al., 2009), e, em seguida, faz-se a classificação mecânica da madeira com equipamentos capazes de quantificar adequadamente o módulo de elasticidade das peças.

De acordo com o projeto de revisão da NBR 7190 (1997), o intuito da classificação visual e mecânica não é separar as peças de melhor qualidade, desprezandose as de qualidade inferior e, sim, conhecer os defeitos e propriedades mecânicas de cada peça, possibilitando seu uso adequado e seguro nas diversas aplicabilidades da construção civil.

Atualmente, há diversas técnicas de ensaios não destrutivos aplicáveis à classificação de peças estruturais de madeira, a exemplo da classificação visual, do ultrassom, da vibração transversal e da vibração longitudinal. Na técnica de vibração transversal, a frequência de vibração transversal do material é associada à rigidez na flexão (CARREIRA e CANDIAN, 2008). Na técnica de vibração longitudinal, a frequência de vibração longitudinal do material também é associada à rigidez na flexão (PITER et al., 2004). Os métodos de ensaios não destrutivos citados estão se tornando cada vez mais relevantes, uma vez que são capazes de oferecer respostas rápidas e correlações lineares elevadas (em média, da ordem de $\mathrm{R}^{2}$ igual a 0,8 ), e o custo dos equipamentos necessários aos ensaios vem caindo ao longo dos últimos anos.

Este trabalho teve como objetivo avaliar a aplicação de métodos de ensaios não destrutivos baseados nas frequências naturais de vibração (ressonâncias) dos modos longitudinal e transversal, comparando-os aos resultados obtidos na flexão estática, para obtenção das propriedades elásticas em peças estruturais de madeira de reflorestamento (Pinus oocarpa e Eucalyptus $s p$.), que são usualmente empregadas na construção civil e habitacional.

\section{MATERIAIS E MÉTODOS}

Neste trabalho foram avaliadas peças de madeira provenientes de florestas plantadas: 24 vigas de Eucalyptus sp. com dimensões nominais de 40 x 60 x 2.000 mm e 14 vigas de Pinus oocarpa com dimensões nominais de $45 \times 90 \times 2.300 \mathrm{~mm}$, ambas sem tratamento; 30 pranchas com dimensões nominais de 40 x $240 \mathrm{x}$ $2.010 \mathrm{~mm}$ e 30 pranchas com dimensões de 40 x 240 x $3050 \mathrm{~mm}$, ambas de Pinus oocarpa e com tratamento preservativo à base de arseniato de cobre cromatado - CCA. Todas as peças de madeira estavam armazenadas em local coberto e já se encontravam em equilíbrio higroscópico com o meio.

\subsection{Ensaio de vibração longitudinal}

Neste ensaio de vibração longitudinal, obteve-se resposta acústica composta por uma ou mais frequências naturais de vibração a partir das quais foi calculado o módulo de elasticidade. O corpo de prova sofre impacto na direção longitudinal, conforme instruções da norma ASTM E1876 (2007), em que a direção do movimento da onda ocorre na mesma direção do modo de vibração longitudinal. Para obtenção das frequências naturais

Revista Árvore, Viçosa-MG, v.36, n.6, p.1155-1161, 2012 
de vibração, foi utilizado o aparelho Sonelastic ${ }^{\circledR}$ Stand Alone, Figura 1, em acordo com as normas ASTM E1876 (2007) e ASTM C215 (2008). O cálculo do módulo de elasticidade devido à primeira frequência de ressonância de vibração longitudinal $E_{1}$ é dado pela Equação 1 .

$$
E_{1}=4 \cdot\left(\frac{m \cdot \mathrm{f}_{1}^{2}}{b}\right) \cdot\left(\frac{l}{h}\right)
$$

sendo: $E_{1}=$ módulo de elasticidade $(\mathrm{MPa}) ; m=$ massa $(\mathrm{kg}) ; \mathrm{f}_{1}=$ frequência de vibração longitudinal $(\mathrm{Hz}) ; b$ $=\operatorname{largura}(\mathrm{mm}) ; h=\operatorname{altura}(\mathrm{mm}) ; l=$ comprimento $(\mathrm{mm})$.

A tecnologia utilizada nos ensaios de vibração longitudinal apresenta um software embarcado em um hardware que faz o processamento do sinal captado pelo microfone, retornando as frequências naturais de vibração. A configuração do sistema consiste em um martelo utilizado para excitar o corpo de prova e um microfone direcional pedestal para a captura de frequências de até $20 \mathrm{kHz}$. O corpo de prova pode ser colocado sobre qualquer superfície, sem que seja necessário suporte específico ou calibrações do sistema. O tempo de preparação, pré-ensaio, é muito pequeno,

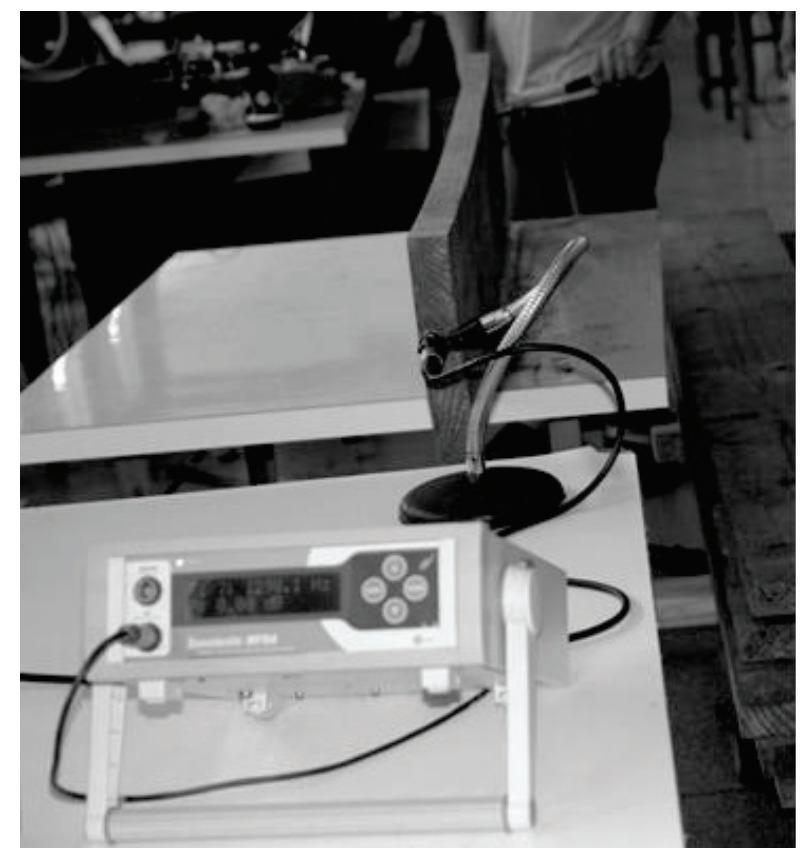

Figura 1 - Equipamento para ensaio de vibração na direção longitudinal - Sonelastic ${ }^{\circledR}$.

Figure 1 - Vibration testing equipment for longitudinal direction - Sonelastic ${ }^{\circledR}$. ou seja, basta ligar o equipamento, posicionar o microfone próximo a uma das extremidades da viga e dar leve pancada com o martelo na extremidade oposta.

\subsection{Ensaio de vibração transversal}

Nos ensaios para determinação dos módulos de elasticidade por meio de vibração transversal foi utilizado o aparelho Metriguard model 340 E-computer. A unidade de interface recebe os sinais provenientes da célula de carga localizada sobre um dos apoios e os transmite para o computador, em que um programa calcula e grava o módulo de elasticidade, a densidade, a frequência de vibração etc. Como procedimento inicial, logo após a montagem do aparelho, faz-se a calibração do sistema, utilizando para isso uma barra de alumínio com as dimensões, massa e módulo de elasticidade conhecidos. Após os ajustes iniciais, a peça a ser ensaiada é colocada sobre os apoios metálicos, e uma liberação é feita no programa, indicando que uma vibração pode ser produzida. Esta pode ser provocada tanto por um simples golpe com a mão quanto também por uma ferramenta manual, por exemplo um martelo. Durante a vibração, é feita uma leitura ou quantas se desejarem, e o programa realiza os cálculos, com os valores expressos diretamente na tela do computador. O cálculo do módulo de elasticidade de vibração transversal $E_{\mathrm{vt}}$ é dado pela Equação 2 .

$$
E_{\mathrm{vt}}=\frac{\mathrm{f}_{\mathrm{r}}^{2} \cdot W \cdot L^{3}}{2,46 \cdot I \cdot g}
$$

sendo: $E_{\mathrm{vt}}=$ módulo de elasticidade dinâmico (MPa); $\mathrm{f}_{\mathrm{r}}=$ frequência de vibração transversal $(\mathrm{Hz}) ; W=$ peso da peça de madeira $(\mathrm{N}) ; L=$ distância entre apoios (m); $I=$ momento de inércia da seção transversal $\left(\mathrm{m}^{4}\right)$; $g=$ aceleração da gravidade $\left(9,8 \mathrm{~m} / \mathrm{s}^{2}\right)$.

\subsection{Ensaio de flexão estática}

Foram realizados ensaios de flexão estática nas peças de madeira com o objetivo de obter o módulo de elasticidade à flexão. $\mathrm{O}$ ensaio foi feito em três pontos nas peças de madeira, conforme a norma ASTM D 198 (2009). O deslocamento foi medido por meio de relógio comparador, resolução $0,01 \mathrm{~mm}$ e curso de $50 \mathrm{~mm}$, na face oposta à superfície de aplicação da força até atingir um limite (L/200), conforme ABNT NBR 7190 (1997). A força foi medida por meio de um anel dinamométrico de capacidade igual a 4,7 kN. O módulo de elasticidade à flexão $E_{\mathrm{M}}$ é dado pela Equação 3 .

Revista Árvore, Viçosa-MG, v.36, n.6, p.1155-1161, 2012 


$$
E_{\mathrm{M}}=\frac{P \cdot L^{3}}{48 \cdot \delta \cdot I}
$$

sendo: $E_{\mathrm{M}}=$ módulo de elasticidade à flexão $(\mathrm{MPa})$; $P=$ incremento de força $(\mathrm{N}) ; L=$ distância entre apoios $(\mathrm{mm}) ; \delta=$ deslocamento vertical devido à força aplicada $(\mathrm{mm}) ; I=$ momento de inércia da seção transversal $\left(\mathrm{mm}^{4}\right)$.

\section{RESULTADOS}

Na Tabela 1 são apresentados os resultados dos módulos de elasticidade das espécies de madeira obtidos por meio das técnicas dos ensaios de vibração longitudinal, vibração transversal e flexão estática. Nessa tabela, as médias dos módulos de elasticidade seguidas de pelo menos uma mesma letra minúscula em cada linha não diferem estatisticamente a $5 \%$ de probabilidade, pelo teste de Tukey.

Com base nos resultados foi possível calcular a porcentagem média de variação de $E_{1}$ e $E_{\mathrm{vt}}$ em relação a $E_{\mathrm{M}}$, por meio das Equações 4 e 5 , respectivamente, conforme apresentado na Tabela 2. Esse procedimento foi adotado por Sales et al. (2010), cujos dados analisados foram obtidos por meio de ultrassom, vibração transversal e flexão estática.

$$
\begin{aligned}
& \text { Porcentagem de variação }(\%)=\frac{E_{1}-E_{\mathrm{M}}}{E_{\mathrm{M}}} \\
& \text { Porcentagem de variação }(\%)=\frac{E_{\mathrm{vt}}-E_{\mathrm{M}}}{E_{\mathrm{M}}}
\end{aligned}
$$

Na Figura 2, têm-se as estimativas dos parâmetros a partir da regressão linear entre os resultados dos ensaios de vibração longitudinal e flexão estática.
Na Figura 3, têm-se as estimativas dos parâmetros a partir da regressão linear entre os resultados dos ensaios de vibração transversal e flexão estática.

\section{DISCUSSÃO}

Observa-se, pela Tabela 1, que entre os valores médios de $E_{1}$ x $E_{\mathrm{vt}}$ e entre os valores médios de $E_{1}$ $\mathrm{x} E_{\mathrm{M}}$ não existe diferença significativa $(\mathrm{P}>0,05)$ para todas as médias dos tratamentos das espécies analisadas. Entre os valores médios de $E_{\mathrm{vt}}$ x $E_{\mathrm{M}}$ não existe diferença significativa $(\mathrm{P}>0,05)$ apenas para a média do tratamento da espécie Pinus oocarpa (45 x $90 \times 2.300 \mathrm{~mm}$ ).

Verifica-se também, pela Tabela 1, que os valores médios de $E_{\mathrm{M}}$ são sempre menores que os valores de $E_{1}$ e $E_{\mathrm{vt}}$ para todas as espécies analisadas. Para os valores médios de $E_{1}$ e $E_{\mathrm{v}}$, não é possível dizer que um deles seja sempre maior do que o outro, uma vez que para a espécie Pinus oocarpa $(45 \times 90 \times 2.300 \mathrm{~mm}) E_{\mathrm{l}}>E_{\mathrm{vt}}$ e, para as outras espécies $E_{1}<E_{\mathrm{vt}}$.

Observam-se, pela Tabela 2, porcentagens médias de variação igual a $13,73 \%$, utilizando a técnica de vibração longitudinal e $15,58 \%$, usando a técnica de vibração transversal. Nota-se que os coeficientes angulares das retas de regressão linear mostrados na Figura 2 indicam um ajuste médio de $91,0 \%$ obtido por meio do ensaio de vibração longitudinal na avaliação não destrutiva de peças de madeira. Já os coeficientes angulares das retas de regressão linear mostrados na Figura 3 apontam ajuste médio de $81,0 \%$ obtido por meio do ensaio de vibração transversal na avaliação não destrutiva de peças de madeira.

\begin{tabular}{|c|c|c|c|c|c|}
\hline Espécies & & $E_{1}(\mathrm{MPa})$ & $E_{\mathrm{vt}}(\mathrm{MPa})$ & $E_{\mathrm{M}}(\mathrm{MPa})$ & $\rho_{\mathrm{ap}}\left(\mathrm{g} / \mathrm{cm}^{3}\right)$ \\
\hline Eucalyptus sp. & Média & $17.147 \mathrm{ab}$ & 18.808 a & $16.368 \mathrm{~b}$ & 0,872 \\
\hline$(40 \times 60 \times 2.000 \mathrm{~mm})$ & $\mathrm{CV}(\%)$ & 16,4 & 15,1 & 16,3 & 16,9 \\
\hline Pinus oocarpa & Média & $10.455 \mathrm{a}$ & $10.304 \mathrm{a}$ & $9.443 \mathrm{a}$ & 0,531 \\
\hline$(45 \times 90 \times 2.300 \mathrm{~mm})$ & $\mathrm{CV}(\%)$ & 28,8 & 28,2 & 29,3 & 7,5 \\
\hline Pinus oocarpa & Média & $14.809 \mathrm{ab}$ & $15.408 \mathrm{a}$ & $13.118 \mathrm{~b}$ & 0,612 \\
\hline$(40 \times 240 \times 2.010 \mathrm{~mm})$ & $\mathrm{CV}(\%)$ & 19,6 & 17,7 & 20,0 & 11,8 \\
\hline Pinus oocarpa & Média & $14.142 \mathrm{ab}$ & $14.614 \mathrm{a}$ & $13.185 \mathrm{~b}$ & 0,602 \\
\hline$(40 \times 240 \times 3.050 \mathrm{~mm})$ & $\mathrm{CV}(\%)$ & 16,0 & 12,4 & 14,6 & 9,4 \\
\hline
\end{tabular}

Tabela 1 - Estatística descritiva dos dados das propriedades mecânicas das peças de madeira.

Table 1 -Descriptive statistics of the mechanical properties of the timber.

sendo: $E_{1}=$ módulo de elasticidade obtido por vibração longitudinal, $E_{\mathrm{vt}}=$ módulo de elasticidade obtido por vibração transversal, $E_{\mathrm{M}}$ $=$ módulo de elasticidade obtido por flexão estática, $\mathrm{CV}=$ coeficiente de variação, $\rho_{\mathrm{ap}}=$ densidade aparente, e as médias seguidas pela mesma letra minúscula não apresentam diferença entre si (Tukey, $\alpha=5 \%$ ).

Revista Árvore, Viçosa-MG, v.36, n.6, p.1155-1161, 2012 
**Significativo ao nível de $1 \%$ de probabilidade.
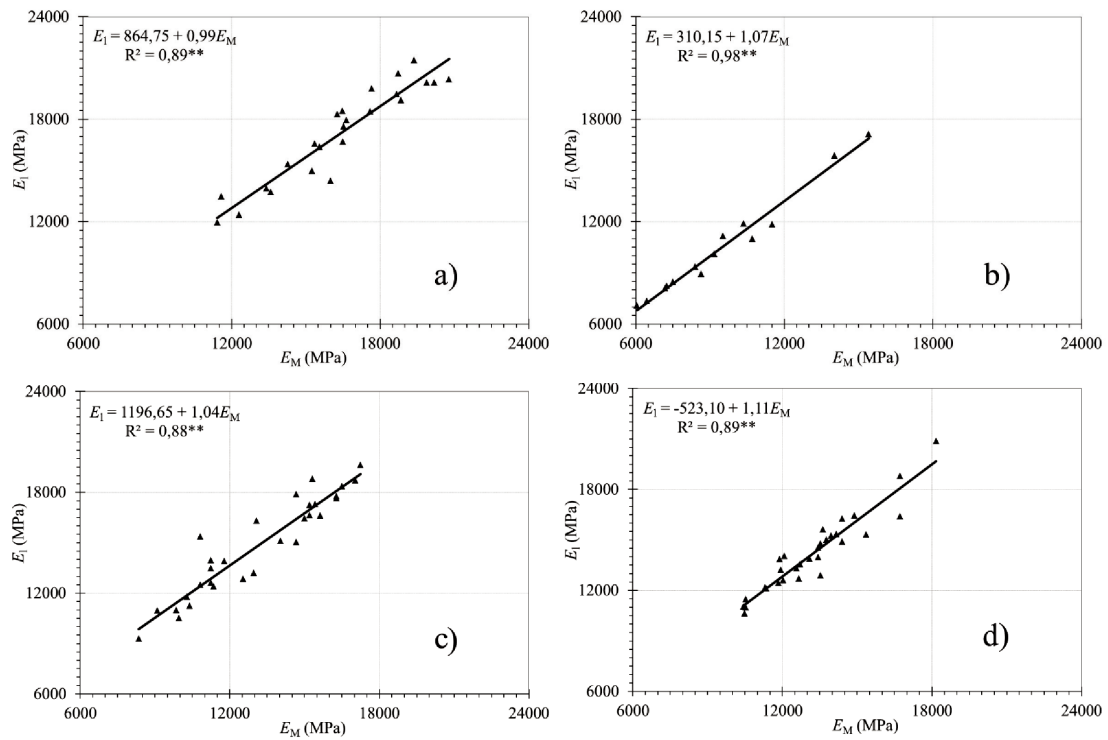

Figura 2 - Relação entre os módulos de elasticidade: a) $E_{1} \times E_{\mathrm{M}}-$ Eucalyptus sp. (40 x $\left.60 \times 2.000 \mathrm{~mm}\right)$, b) $E_{1} \times E_{\mathrm{M}}-$ Pinus oocarpa $(45 \times 90 \times 2.300 \mathrm{~mm})$, c) $E_{1} \times E_{\mathrm{M}}$ - Pinus oocarpa $(40 \times 240 \times 2.010 \mathrm{~mm}) \mathrm{e}$ d) $E_{1} \times E_{\mathrm{M}}-$ Pinus oocarpa (40 × $240 \times 3.050 \mathrm{~mm})$.

Figure 2 - Relationship between modulus of elasticity: a) $E_{l} \times E_{M}$ - Eucalyptus sp. $\left.(40 \times 60 \times 2.000 \mathrm{~mm}), b\right) E_{l} \times E_{M}$ Pinus oocarpa $(45 \times 90 \times 2.300 \mathrm{~mm}), c) E_{l} \times E_{M}$-Pinus oocarpa $(40 \times 240 \times 2.010 \mathrm{~mm})$ e d) $E_{l} \times E_{M}-$ Pinus oocarpa $(40 \times 240 \times 3.050 \mathrm{~mm})$.

**Significativo ao nível de $1 \%$ de probabilidade.
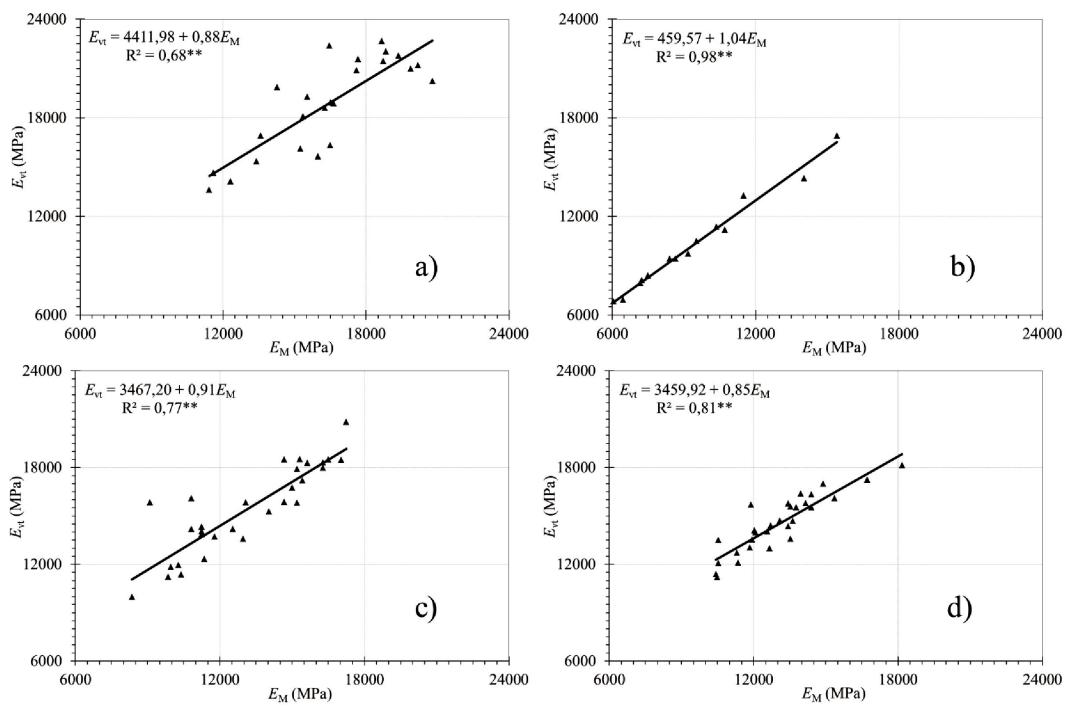

Figura 3 - Relação entre os módulos de elasticidade: a) $\mathrm{E}_{\mathrm{vt}} \times \mathrm{E}_{\mathrm{M}}-$ Eucalyptus sp. $(40 \times 60 \times 2.000 \mathrm{~mm})$, b) $\mathrm{E}_{\mathrm{vt}} \times \mathrm{E}_{\mathrm{M}}$ Pinus oocarpa $(45 \times 90 \times 2.300 \mathrm{~mm})$, c) $\mathrm{E}_{\mathrm{vt}} \times \mathrm{E}_{\mathrm{M}}$ - Pinus oocarpa $(40 \times 240 \times 2.010 \mathrm{~mm}) \mathrm{e}$ ) $\mathrm{E}_{\mathrm{vt}} \times \mathrm{E}_{\mathrm{M}}^{\mathrm{vt}}$ - Pinus oocarpa ( $40 \times 240 \times 3.050 \mathrm{~mm})$.

Figure 3 -Relationship between modulus of elasticity: a) $E_{v t} \times E_{M}$-Eucalyptus sp. $\left.(40 \times 60 \times 2.000 \mathrm{~mm}), b\right) E_{v t} \times E_{M}$ - Pinus oocarpa $(45 \times 90 \times 2.300 \mathrm{~mm}), c) E_{v t} \times E_{M}$-Pinus oocarpa $\left.(40 \times 240 \times 2.010 \mathrm{~mm}) \mathrm{e} d\right) E_{v t} \times E_{M}{ }^{-}$ Pinus oocarpa $(40 \times 240 \times 3.050 \mathrm{~mm})$. 
Tabela 2 - Porcentagem média de variação. Table 2 -Average percent variation.

\begin{tabular}{lcc}
\hline \multirow{2}{*}{ Espécies } & \multicolumn{2}{c}{$\begin{array}{c}\text { Porcentagem média } \\
\text { de variação }(\%)\end{array}$} \\
\cline { 2 - 3 } & $\begin{array}{c}\text { Vibração } \\
\text { longitudinal }\end{array}$ & $\begin{array}{c}\text { Vibração } \\
\text { transversal }\end{array}$ \\
\hline $\begin{array}{l}\text { Eucalyptus sp. } \\
(40 \times 60 \times 2.000 \mathrm{~mm})\end{array}$ & 4,89 & 15,58 \\
$\begin{array}{l}\text { Pinus oocarpa } \\
(45 \times 90 \times 2.300 \mathrm{~mm})\end{array}$ & 11,08 & 9,46 \\
Pinus oocarpa & 13,73 & 15,33 \\
$(40 \times 240 \times 2.010 \mathrm{~mm})$ & 10,62 & 10,23 \\
Pinus oocarpa & & \\
$(40 \times 240 \times 3.050 \mathrm{~mm})$ & & \\
\hline
\end{tabular}

Verificou-se que o método de ensaio utilizando a técnica de vibração longitudinal apresentou valores fortemente correlacionados (valores de $\mathrm{R}^{2}$ próximos de 1,0). O método de ensaio utilizando a técnica de vibração transversal caracterizou-se por apresentar valores menos correlacionados, distanciando de 1,0. Oliveira (2006), empregando o método de ensaio não destrutivo de ultrassom, constatou que é necessário adequar a frequência do equipamento com o tamanho do corpo de prova, para que se obtenha a correta velocidade ultrassônica em madeiras. Neste estudo, observou-se que é necessário o corpo de prova ser três vezes maior que o comprimento de onda.

\section{CONCLUSÃO}

Os resultados dos módulos de elasticidade obtidos por meio dos três métodos de ensaios - vibração longitudinal, vibração transversal e flexão estática resultaram em valores próximos e apresentaram correlação significativa a $1 \%$ de probabilidade. Concluiu-se que, dada a proximidade dos resultados alcançados pelo ensaio de vibração longitudinal e aqueles obtidos pelos já consolidados ensaios de vibração transversal e flexão estática, pode-se afirmar que o ensaio de vibração longitudinal responde com boa precisão aos ensaios de caracterização dos módulos de elasticidade de vigas de madeira. Sugere-se, assim, que a escolha entre um ou outro método seja feita, também, pela praticidade.

A particularidade do método de ensaio da vibração longitudinal em obter a resposta acústica do corpo de prova, empregando-se um microfone e dispensando a utilização de um suporte específico para o corpo de prova e calibrações prévias do equipamento, facilita os ensaios e reduz significativamente o tempo de caracterização, principalmente quando se leva em conta sua aplicação, em grande escala, para classificação de peças estruturais de madeiras nas diversas aplicações da construção civil.

\section{REFERÊNCIAS}

\section{AMERICAN SOCIETY FOR TESTING AND \\ MATERIALS - ASTM. ASTM C215: Standard test method for fundamental transverse, longitudinal and torsional frequencies of concrete specimens. Philadelphia: 2008.}

\section{AMERICAN SOCIETY FOR TESTING AND}

MATERIALS - ASTM. ASTM D198: Standard

Test Methods of Static Tests of Lumber in Structural Sizes. Philadelphia: 2009.

\section{AMERICAN SOCIETY FOR TESTING AND MATERIALS - ASTM. ASTM E1876:}

Standard test method for dynamic Young's modulus, shear modulus and Poisson's ratio by Impulse Excitation of Vibration. Philadelphia: 2007.

\section{ASSOCIAÇÃO BRASILEIRA DE NORMAS TÉCNICAS - ABNT. NBR 7190: Projeto de estruturas de madeira. Rio de Janeiro: 1997.}

CARREIRA, M. R.; CANDIAN, M. Teste de um equipamento para classificação de peças estruturais de madeira pela técnica da vibração transversal. Semina: Ciências Exatas e da Terra, v.29, n.1, p. 3-14, 2008.

CARREIRA, M. R.; DIAS, A. A. Classificação visual de coníferas: análise da aplicação do método norte-americano às espécies de Pinus spp. plantadas no Brasil. Scientia Forestalis, n.67, p.78-87, 2005.

FIORELLI, J.; DIAS, A. A.; COIADO, B. Propriedades mecânicas de peças com dimensões estruturais de Pinus sp: correlação entre resistência à tração e classificação visual. Revista Árvore, v.33, n.4, p.741-750, 2009.

OLIVEIRA, F. G. R. et al. Efeito do comprimento do corpo-de-prova na velocidade ultra-sônica em madeiras. Revista Árvore, v.30, n.1, p. 141-145, 2006. 
PITER, J. C.; ZERBINO, R. L.; BLAB, H. J.

Effectiveness of fundamental resonant frequency for determining the elastic properties of Argentinean Eucalyptus grandis in structural sizes. Holz Roh Werkst, v.62, n.2, p.88-92, 2004.
SALES, A.; CANDIAN, M.; SALLES CARDIN, V. Nondestructive evaluation of timber: the new Brazilian code for the design of timber structures. Materials and Structures, v.43, p.213-221, 2010. 
